\title{
Vulvar Acquired Melanocytic Nevus
}

National Cancer Institute

\section{Source}

National Cancer Institute. Vulvar Acquired Melanocytic Nevus. NCI Thesaurus. Code C40331.

A nevus that arises from the vulva during childhood and grows with increasing age. 